doi:10.1017/\$1041610219001443

\section{Depression in older Medicare beneficiaries with hearing impairment by sex, race/ethnicity and insurance status}

Hearing impairment is associated with cognitive impairment (e.g. cognitive decline and dementia) and lower use of health services in older adults (Amieva et al. 2015; Mahmoudi et al. 2018). Hearing impairment may also be more significantly associated with depression among older adults (Mener et al. 2013). Using the most recent data from 2016 Medicare Current Beneficiary Survey (MCBS), which captures nationally representative trends of physical and mental health status and health services use among Medicare beneficiaries (Centers for Medicare and Medicaid Services. Medicare Current Beneficiary Survey (MCBS) 2019), I investigated prevalence of depression by key demographic factors (i.e. sex, race/ethnicity and insurance status) and the factors associated with depression in older adults with hearing impairment.

The following three questionnaire items were asked to Medicare beneficiaries to assess hearing impairment: using a hearing aid (yes or no); selfreported hearing (no trouble, a little trouble or a lot of trouble); and difficulty of communicating with a doctor because of hearing (no trouble, a little trouble or a lot of trouble). I constructed hearing impairment variable, where "yes" indicates "yes" or "a lot of trouble" and "no" indicates otherwise. In the final analytic sample, I included Medicare beneficiaries who are 65 years or older with hearing impairment ( $n=5,519$ unweighted). Survey participants were also asked whether they ever had depression (yes or no).

When extrapolated from the survey sample, about 21.5 million older Medicare beneficiaries $(47.6 \%)$ were identified with hearing impairment. The majority of the sample were aged 65-74 $(52.7 \%)$, male $(52.7 \%)$, and non-Hispanic white $(82.8 \%)$. Of older Medicare beneficiaries with hearing impairment, $23.4 \%$ (5.0 million) had depression. The prevalence of depression varied by sex, race/ethnicity, and insurance status $(p<0.05)$ (see Figure 1).
In the multivariable-adjusted analysis (see Table 1), being female and dual-eligible were associated with a higher likelihood of being depressed than male and Medicare-only counterparts, respectively (adjusted odds ratio $[\mathrm{AOR}]=1.98 ; 95 \%$ confidence intervals $[\mathrm{CI}]=1.70,2.31$ for sex, and $\mathrm{AOR}=2.09 ; 95 \%$ $\mathrm{CI}=1.58,2.78$ for insurance status). In race/ethnicity, those with Hispanic identity had a higher rate of depression than non-Hispanic whites, but this pattern was no longer significant when adjusted for other demographic factors.

Overall, nearly one of four older Medicare beneficiaries with hearing impairment had depression, and the prevalence of depression varied by key demographic factors. Healthcare providers should recognize that depression is not only common, but patients of certain demographic background may be particularly vulnerable for depression. For instance, dualeligibles often face lower socio-economic status and/or multi-morbidities than those with Medicare only (Feng 2018). Thus, these individuals may need patient-centered, coordinated care to improve both quality of care and quality of life outcomes.

Some limitations deserve comment from this study. First, MCBS is a self-reported survey, and hearing impairment and/or depression may not be based on clinical definitions. Second, no causation can be made due to the cross-sectional nature of the study. Despite these limitations, the current study highlights that depression is common among older Medicare beneficiaries with hearing impairment, and females and dual-eligibles are particularly associated with a higher likelihood of being depressed than males and those with Medicare only, respectively. Patient-oriented research is needed to address depression in these vulnerable older adults.

\section{Funding/support and role of the funder/sponsor}

In the past three years, the author received funding support from the National Institutes of Health (NIH) (\#T32AG019134) through Yale University School of Medicine. The funding agency, NIH, had no role on this intellectual contribution from the study conception to journal publication.

\section{Conflicts of interest}

The author has no conflicts of interest to declare. 
Table 1. Demographic characteristics (column \%) and multivariable-adjusted logistic regression analyses to determine factors associated with depression in older Medicare beneficiaries with hearing impairment

\begin{tabular}{|c|c|c|c|c|c|}
\hline & \multicolumn{3}{|c|}{ Depression } & \multirow[b]{2}{*}{ Unadjusted odds ratio } & \multirow[b]{2}{*}{ Adjusted odds ratio } \\
\hline & No $(\%)$ & Yes $(\%)$ & Total (\%) & & \\
\hline \multicolumn{6}{|l|}{ Sample size } \\
\hline Unweighted & 4,280 & 1,239 & 5,519 & & \\
\hline Weighted & $16,450,696$ & $5,036,340$ & $21,487,036$ & & \\
\hline \multicolumn{6}{|l|}{ Age } \\
\hline $65-74$ & 51.2 & 57.5 & 52.7 & Reference & Reference \\
\hline$\geq 75$ & 48.8 & 42.5 & 47.3 & $0.77(0.67,0.90)$ & $0.72(0.61,0.84)$ \\
\hline \multicolumn{6}{|l|}{ Sex } \\
\hline Male & 56.8 & 39.2 & 52.7 & Reference & Reference \\
\hline Female & 43.2 & 60.8 & 47.3 & $2.04(1.76,2.37)$ & $2.05(1.75,2.39)$ \\
\hline \multicolumn{6}{|l|}{ Race/ethnicity } \\
\hline Non-Hispanic white & 83.3 & 81.1 & 82.8 & Reference & Reference \\
\hline Non-Hispanic black & 6.0 & 5.1 & 5.8 & $0.87(0.62,1.24)$ & $0.70(0.48,1.03)$ \\
\hline Hispanic & 4.8 & 7.4 & 5.4 & $1.58(1.20,2.10)$ & $1.33(0.99,1.79)$ \\
\hline Other & 5.9 & 6.5 & 6.0 & $1.12(0.80,1.58)$ & $0.98(0.68,1.42)$ \\
\hline \multicolumn{6}{|l|}{ Dual-eligibility } \\
\hline No & 95.1 & 89.3 & 93.7 & Reference & Reference \\
\hline Yes & 4.9 & 10.7 & 6.3 & $2.32(1.78,3.03)$ & $2.10(1.59,2.77)$ \\
\hline
\end{tabular}

Note: Data are from 2016 Medicare Current Beneficiary Survey (MCBS). All analyses were conducted using Stata MP/6-Core version 15.1 (College Station, TX), accounting for complex survey design and sampling weights used in the MCBS.

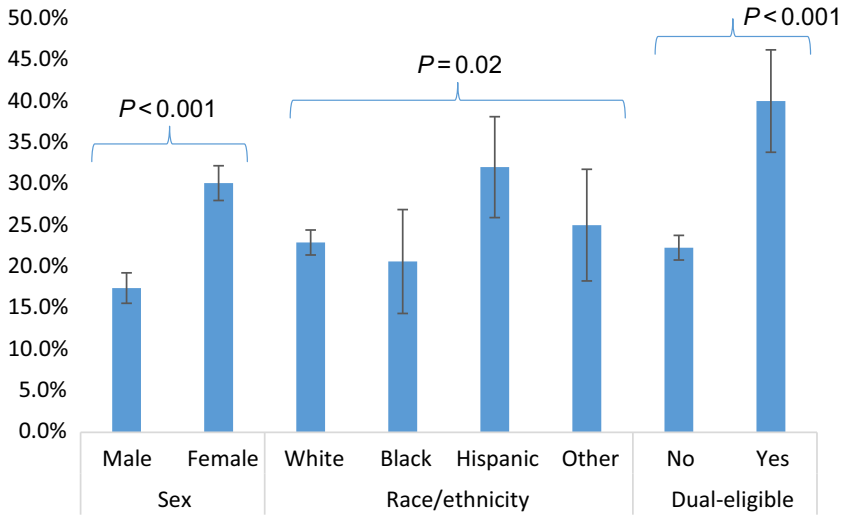

Figure 1. Prevalence of depression in older Medicare beneficiaries with hearing impairment by sex, race/ethnicity and insurance status. Note: Data are from 2016 Medicare Current Beneficiary Survey (MCBS). All analyses were conducted using Stata MP/6-Core version 15.1 (College Station, TX), accounting for complex survey design and sampling weights used in the MCBS. $P$-values were calculated from weight-corrected Pearson's chi-squared statistics.

\section{Compliance with ethical standards}

This article does not contain any studies with human participants or animals performed by the authors. All research procedures performed in this study are in accordance with the ethical standards of the Institutional Review Board at University of Connecticut School of Medicine and Yale University School of Medicine.

\section{References}

Amieva, H., Ouvrard, C., Giulioli, C., Meillon, C., Rullier, L. and Dartigues, J.F. (2015). Self-reported hearing loss, hearing aids, and cognitive decline in elderly adults: A 25-year study. Fournal of the American Geriatrics Society, 63, 2099-2104.

Centers for Medicare and Medicaid Services. Medicare Current Beneficiary Survey (MCBS). (2019). https:// www.cms.gov/research-statistics-data-and-systems/ research/mcbs/; last accessed June 26, 2019.

Feng, Z. (2018). Dual eligibles: who are they and why are they important? Public Policy \& Aging Report, 28, 56-63.

Mahmoudi, E., Zazove, P., Meade, M. and McKee, M.M. (2018). Association between hearing aid use and health care use and cost among older adults with hearing loss. $\mathcal{F} A M A$ Otolaryngology-Head E Neck Surgery, 144, 498-505.

Mener, D.J., Betz, J., Genther, D.J., Chen, D. and Lin, F.R. (2013). Hearing loss and depression in older adults. Fournal of the American Geriatrics Society, 61, 1627-1629.

TAeho Greg RHEE ${ }^{1,2}$ (1)

${ }^{1}$ Department of Community Medicine and Health Care, School of Medicine, University of Connecticut, Farmington, CT, USA

${ }^{2}$ Department of Psychiatry, School of Medicine, Yale University, New Haven, CT, USA

Correspondence should be addressed to: Greg Rhee, $\mathrm{PhD}$, Department of Community Medicine and Health Care, School of Medicine, University of Connecticut Health Center, 263 Farmington Ave, Farmington CT, 06530, USA. Phone: +1-773-398-9564. Email: rhee@uchc.edu; tgrhee.research@gmail.com 